\title{
Perfil de saúde física e mental de homens e mulheres privados de liberdade: um estudo comparativo
}

Physical and mental health profile of men and women deprived of their liberty: a comparative study

Perfil de salud física y mental de hombres y mujeres privados de libertad: un estudio comparativo

Joicielly França Bispo¹, Thaciana de Melo Monte Pedrosa1, Flaviane Maria Pereira Belo², Layanne Almeida Cezário ${ }^{1}$, Adriana de Lima Mendonça1, Alba Maria Bomfim de França ${ }^{1}$, Givânya Bezerra de Melo ${ }^{1 *}$.

\section{RESUMO}

Objetivo: Comparar o perfil de saúde física e mental de homens e mulheres privados de liberdade. Métodos: Trata-se de estudo transversal, com abordagem quantitativa, que entrevistou 128 pessoas privadas de liberdade de ambos os sexos, em duas unidades prisionais de Alagoas. Os dados foram analisados através do software IBM SPSS Statistics. A pesquisa foi aprovada pelo Comitê de Ética em Pesquisa. Resultados: Na comparação por sexos, a prevalência de queixas/problemas clínicos foi mais acentuada entre as mulheres $(62,3 \%)$ do que entre os homens $(39,2 \%)$. A prevalência de tratamento de saúde mental referido entre as mulheres foi de $51,9 \%$, superando em duas vezes a encontrada nos homens $(21,6 \%)$. Verificou-se que $24,7 \%$ das mulheres e $13,7 \%$ dos homens possuíam sintomas de ansiedade. Insônia e depressão foram referidas por $15,6 \%$ das mulheres, enquanto $13,7 \%$ dos homens apresentaram queixas de insônia e $2 \%$ de depressão. Também houve maior prevalência do uso de psicofármacos entre as mulheres (55,8\% vs. $33,3 \%$ entre os homens). Conclusão: Homens e mulheres em situação de cárcere apresentaram prevalências acentuadas de problemas físicos e mentais, mais elevados, entretanto, entre as mulheres. Esses resultados são relevantes para o planejamento e a atenção à saúde desse público.

Palavras-chave: Saúde, Saúde mental, Pessoas privadas de liberdade, Homens, Mulheres.

\begin{abstract}
Objective: Comparison of the physical and mental health profile of men and women deprived of liberty. Methods: This is a cross-sectional study, with a quantitative approach, which interviewed 128 people deprived of liberty of both sexes, in two prisons in Alagoas. Data were through IBM SPSS Statistics software. The research was approved by the Research Ethics Committee. Results: When comparing genders, the prevalence of clinical complaints/problems was more pronounced among women (62.3\%) than among men (39.2\%). The prevalence of self-reported mental health treatment among women was $51.9 \%$, twice that found among men (21.6\%). It was found that $24.7 \%$ of women and $13.7 \%$ of men had anxiety symptoms. Insomnia and depression were formed by $15.6 \%$ of women, while $13.7 \%$ of men were deaf from insomnia and $2 \%$ from depression. There was also a higher prevalence of psychoactive drug use among women $(55.8 \%$ vs. $33.3 \%$ among men). Conclusion: Men and women in prison have higher prevalences of medical and mental problems, which are higher, however, among women. These results are relevant for the planning and health care of this public.
\end{abstract}

Keywords: Health, Mental health, People deprived of liberty, Men, Women.

${ }^{1}$ Centro Universitário Tiradentes (Unit), Maceió - AL. *E-mail: givanya@gmail.com

2 Universidade Federal de Alagoas (Ufal), Maceió - AL.

O estudo faz parte do Programa de Iniciação Científica do Centro Universitário Tiradentes de Maceió/ Alagoas/Brasil (PIBIC-Unit).

SUBMETIDO EM: 7/2021

ACEITO EM: 8/2021

PUBLICADO EM: 9/2021 


\section{RESUMEN}

Objetivo: Comparación del perfil de salud física y mental de hombres y mujeres privados de libertad. Métodos: Se trata de un estudio transversal, con enfoque cuantitativo, que entrevistó a 128 personas privadas de libertad de ambos sexos, en dos cárceles de Alagoas. Los datos se obtuvieron a través del software IBM SPSS Statistics. La investigación fue aprobada por el Comité de Ética en Investigación. Resultados: Al comparar los géneros, la prevalencia de quejas / problemas clínicos fue más pronunciada entre las mujeres $(62,3 \%)$ que entre los hombres $(39,2 \%)$. La prevalencia de tratamiento de salud mental autoinformada entre las mujeres fue del $51,9 \%$, el doble de la encontrada entre los hombres $(21,6 \%)$. Se encontró que el $24,7 \%$ de las mujeres y el $13,7 \%$ de los hombres presentaban síntomas de ansiedad. El insomnio y la depresión estaban formados por el $15,6 \%$ de las mujeres, mientras que el $13,7 \%$ de los hombres eran sordos por insomnio y el $2 \%$ por depresión. También hubo una mayor prevalencia de consumo de drogas psicoactivas entre las mujeres (55,8\% frente al 33,3\% entre los hombres). Conclusión: Los hombres y mujeres en prisión tienen una mayor prevalencia de problemas médicos y mentales, que son más altos, sin embargo, entre las mujeres. Estos resultados son relevantes para la planificación y la atención de la salud de este público.

Palabras clave: Salud, Salud mental, Personas privadas de libertad, Hombres, Mujeres.

\section{INTRODUÇÃO}

O aumento do encarceramento é um fenômeno preocupante, tendo em vista que mais de 11 milhões de pessoas privadas de liberdades encontram-se reclusas nas penitenciárias do mundo (WORLD PRISON BRIEF, 2018). A população carcerária brasileira no primeiro semestre de 2020 era de 753.966 reclusos, sendo 36.999 mulheres e 716.967 homens. Durante o mesmo período, Alagoas contava com 7.726 pessoas no presídio do estado (MINISTÉRIO DA JUSTIÇA, 2020).

De acordo com Alves JP, et al. (2017), a população privada de liberdade vivencia condições de iniquidade social e a insatisfatória condição dos serviços de saúde ofertados no sistema penitenciário. A superlotação das celas e a condição de precariedade que o ambiente prisional oferece, torna o local insalubre, aumentando a suscetibilidade para o contágio de doenças que facilmente se proliferam (PEREIRA WRM e FROTA AS, 2020).

Outros aspectos de agravamento dos fatores estruturais dizem respeito à má alimentação, ao sedentarismo, ao uso de drogas, à precariedade da higiene e aos efeitos emocionais relacionados ao aprisionamento (estigma e mortificação da identidade da pessoa condenada), que, somados, geram uma dupla penalização: a pena de prisão propriamente dita e o lamentável estado de saúde que o preso adquire durante sua permanência no cárcere. Faz-se necessária uma assistência em saúde que garanta de fato os princípios de acessibilidade, integralidade, resolutividade e humanização, previstos na Constituição Federal e nas diretrizes do Sistema Único de Saúde (SUS) (PEREIRA WRM e FROTA AS, 2020).

O contexto de crescimento da população carcerária e as complexidades do ambiente prisional têm contribuído para atenção uma à saúde ineficaz, provocando efeitos negativos nas dimensões físicas e psicológicas das pessoas privadas de liberdade (RODRIGUES MIL e CORDAZZO K, 2019). A assistência à saúde é negligenciada, os cuidados ofertados, em sua maioria são limitados, com adversidades para o acesso de qualidade à saúde, permitindo que apenas ocorra uma redução de danos dos problemas mais emergentes (KOLLING GJ, et al., 2013; NETO FJ, et al., 2019; SANTANA JCB e REIS FCA, 2019).

Sabe-se que tal contexto infringe os direitos das pessoas privadas de liberdade, indo de encontro ao Plano Nacional de Saúde, no Sistema Penitenciário (PNSSP), que estabelece um acesso qualificado aos serviços de saúde, com ênfase na promoção e prevenção de agravos (GALVÃO MCB e DAVIM RMB, 2013; MINISTÉRIO DA SAÚDE, 2014). As consequências do déficit da assistência para o atendimento das necessidades específicas desse público de forma integral traduzem-se nas altas prevalências de problemas físicos e mentais. Os desfechos menos favoráveis são mais comuns entre as mulheres, tendo em vista as suas especificidades em relação à vivência do encarceramento e o seu papel social (OLIVEIRA KRV, et al., 2019; MINISTÉRIO DA JUSTIÇA, 2014). 
Quando comparadas aos homens, as mulheres são as mais afetadas pelos problemas físicos e mentais, com maiores chances de serem diagnosticadas com algum tipo de doença crônica (HUGHES MH, et al., 2020). Gottfried ED e Christopher SC (2017) demonstraram que elas tendem a apresentar maior incidência de transtornos mentais; no estudo, $75 \%$ das mulheres e $63 \%$ dos homens possuíam problemas de saúde mental.

Além disso, ocorre uma evidente distinção de gênero sobre as relações familiares mantidas no decorrer do encarceramento, o que provoca grandes repercussões a saúde. As mulheres privadas de liberdade são abandonas por seus cônjuges quando sentenciadas, seja pelo fato de se tornar uma mulher que cometeu um delito ou por seus companheiros criarem outras relações afetivas mais rapidamente. Em contrapartida, as mulheres possuem uma tendência a cuidar da família e a cultivar o relacionamento com seu parceiro, mesmo que este esteja encarcerado (MINAYO MCS e RIBEIRO AP, 2016).

De acordo com Santos MV, et al. (2017), ter saúde envolve uma tríade de bem-estar físico, mental e social, esses fatores possuem uma inter-relação entre si, contribuindo para completa saúde do indivíduo. Contudo, fica evidente que o ambiente penitenciário influencia negativamente na saúde do encarcerado. O que é notório no estudo de Henry BF (2020), no qual $12,6 \%$ das pessoas privadas de liberdade já vivenciaram uma doença mental grave e $25,5 \%$ outros distúrbios de saúde mental por estarem reclusos em um ambiente desumano, que não exerce com magnitude sua real função de ressocialização (BASSANI B e LUCAS CL, 2017).

Diante desse contexto, o presente estudo teve como objetivo comparar o perfil de saúde física e mental de homens e mulheres privados de liberdade.

\section{MÉTODOS}

\section{Desenho do estudo}

O presente estudo faz parte de uma pesquisa maior intitulada: 'Depressão, ansiedade e experiências traumáticas na infância de pessoas privadas de liberdade: um estudo comparativo'. Caracteriza-se como um estudo de corte transversal, com coleta de dados entre dezembro de 2018 e fevereiro de 2020. Foram incluídas duas unidades prisionais de regime fechado: um Núcleo de Ressocialização Masculino e uma unidade prisional feminina de Alagoas/Brasil.

\section{Sujeitos e procedimento para a coleta de dados}

A coleta de dados foi realizada em duas etapas independentes em uma unidade prisional masculina, um Núcleo de Ressocialização, e em uma unidade prisional feminina, que tem o funcionamento de uma unidade prisional convencional. A amostragem foi por conveniência, sendo entrevistados homens e mulheres que se encontravam no local, no dia da coleta de dados. Foram critérios de elegibilidade: possuir no mínimo 18 anos; estar no mínimo há três meses cumprindo pena em regime fechado na penitenciária. Foram excluídas pessoas com comprometimento clínico, psíquico ou cognitivo que impedisse a compreensão das perguntas e a comunicação na entrevista.

Para o cálculo amostral, considerou-se: nível de confiança de 80\%; erro máximo de estimativa $\alpha=0,2$ e valor crítico $Z \alpha / 2$ = 1,28; tamanho da população no estrato feminino (167 mulheres em janeiro de 2018), tamanho da população no estrato masculino (122 homens em janeiro de 2018) e estimativa de perdas de 10\% (SECRETARIA DE ESTADO DE RESSOCIALIZAÇÃO E INCLUSÃO SOCIAL (SERIS), 2018). Com base nesses parâmetros, o total de entrevistados previstos foi de 128 pessoas, divididos proporcionalmente em 51 homens do Núcleo de Ressocialização da Capital e 77 mulheres da Unidade Prisional Feminina.

\section{Instrumentos}

O instrumento utilizado nesta pesquisa foi um "Formulário Sociodemográfico". Trata-se de um questionário estruturado elaborado pelos próprios autores do estudo e composto por 24 questões contendo variáveis sociodemográficas e de saúde mental. 
Para o presente estudo foram utilizadas as seguintes variáveis: problema de saúde atual (sim/não/qual o problema); fez tratamento de saúde mental ( $\operatorname{sim} /$ não); queixa de saúde mental atual (sim/não/qual queixa); uso atual de algum medicamento controlado ( $\mathrm{sim} /$ não/qual medicamento/tempo de uso do medicamento).

A variável "problema de saúde atual" foi avaliada a partir do relato dos entrevistados no período atual de encarceramento. Para fins de análise, os problemas foram classificados, posteriormente, pelo principal sistema corporal afetado. A variável "tratamento de saúde mental anterior" refere-se ao período pregresso ao encarceramento. A variável "queixa de saúde mental atual" foi investigada para o período de reclusão e incluiu as queixas ou os "diagnósticos" referidos pelos entrevistados. As variáveis "uso de psicofármaco atual" e "tempo de uso de psicofármacos" referem-se ao uso dos psicofármacos de forma controlada, fornecidos no sistema prisional no período do encarceramento. Os psicofármacos apontados foram apresentados de acordo com a classe principal que integram (SENA EP, et al., 2011).

\section{Procedimento para análise dos dados}

Os dados da pesquisa foram digitados por dois pesquisadores independentes no Microsoft Excel® versão 2016. Após comparação e correções necessárias, foram exportados para o software IBM Statistical Package for the Social Sciences (S.P.S.S.) for Windows versão 22.0, versão 21. Na análise dos dados, aplicou-se a estatística descritiva com o cálculo de frequências (variáveis qualitativas) e médias como medida de tendência central (variáveis quantitativas).

Para calcular as diferenças nas distribuições entre os grupos de homens e mulheres, aplicou-se o teste $X^{2}$ (qui-quadrado) e o teste T na comparação de médias (amostras independentes). No X², H0 foi assumida para igualdade das proporções das variáveis qualitativas entre os grupos (masculino e feminino); e H1 na diferença dessas proporções entre os grupos. No teste $\mathrm{T}, \mathrm{H} 0$ foi assumida na igualdade das médias dos grupos e $\mathrm{H} 1$ na ocorrência de diferença. Foi aplicada significância estatística $p<0,05$ para estabelecer a diferença entre 0 grupo feminino e o grupo masculino nas variáveis estudadas. Todas as análises foram realizadas com $95 \%$ de confiança.

\section{Aspectos Éticos}

Este estudo integra o programa de iniciação científica do Centro Universitário Tiradentes. Ele foi aprovado pelo Comitê de Ética em Pesquisa da mesma instituição segundo os pareceres de no 3.539.447, no 3.539.450. Todos os aspectos da Resolução 466/2012 do Conselho Nacional de Saúde do Ministério da Saúde do Brasil (2012) foram seguidos e as entrevistas foram autorizadas pelos entrevistados por meio da assinatura/impressão datiloscópica do Termo de Consentimento Livre e Esclarecido.

\section{RESULTADOS}

Neste estudo, das 77 mulheres estudadas, 48,7\% possuíam entre 18 a 29 anos, 63,6\% declararam-se pretas ou pardas e 77,9\% estavam reclusas pela primeira vez. Em relação aos 51 homens estudados, $78,4 \%$ tinham 30 anos ou mais, $60,8 \%$ se autodeclararam pretos ou pardos e estavam reclusos pela primeira vez. No que se refere ao grau de escolaridade, $68,8 \%$ das mulheres cursaram a alfabetização e $37,3 \%$ dos homens concluíram o ensino médio, contudo, $66,2 \%$ das mulheres não estavam estudando no período de reclusão e $34 \%$ dos homens estavam cursando o ensino médio.

Dos 128 homens e mulheres privados de liberdade, verificou-se diferença estatística significativa para problemas clínicos no momento da entrevista $(p=0,010)$, tratamento anterior de saúde mental $(p=0,001)$, tempo de duração de tratamento $(p=0,010)$, queixas de problemas de saúde mental atual $(p=0,003)$, uso de psicofármacos $(p=0,012)$ e tempo de tratamento com psicofármacos $(p=0,010)$ (Tabela 1). 
Tabela 1 - Comparação entre o perfil de saúde de mulheres e homens privados de liberdade em duas unidades prisionais de Alagoas - $2020(n=128)$.

\begin{tabular}{|c|c|c|c|}
\hline Variáveis & $\begin{array}{c}\text { Mulheres ( } \mathrm{n}=77) \\
\mathrm{N}(\%)\end{array}$ & $\begin{array}{c}\text { Homens }(n=51) \\
N(\%)\end{array}$ & $p$-valor \\
\hline \multicolumn{4}{|l|}{ Problema clínico (atual) } \\
\hline Sim & $48(62,3)$ & $20(39,2)$ & $0,010^{*}$ \\
\hline Não & $29(37,7)$ & $2(60,8)$ & \\
\hline \multicolumn{4}{|c|}{ Tratamento de saúde mental anterior } \\
\hline Sim & $40(51,9)$ & $11(21,6)$ & $0,001^{*}$ \\
\hline Não & $37(48,1)$ & $40(78,4)$ & \\
\hline \multicolumn{4}{|c|}{ Tempo de tratamento (anos) } \\
\hline Média (DP) & $4,85(6,16)$ & $5,88(10,87)$ & $0,010^{*}$ \\
\hline Mínimo- Máximo & $0-27$ & $0-36$ & \\
\hline \multicolumn{4}{|c|}{ Queixa de saúde mental (atual) } \\
\hline Sim & $38(49,4)$ & $12(23,5)$ & $0,003^{*}$ \\
\hline Não & $39(50,6)$ & $39(76,5)$ & \\
\hline \multicolumn{4}{|c|}{ Uso de psicofármaco (atual) } \\
\hline Sim & $43(55,8)$ & $17(33,3)$ & $0,012^{*}$ \\
\hline Não & $34(44,2)$ & $34(66,7)$ & \\
\hline \multicolumn{4}{|c|}{ Tempo de uso de psicofármacos } \\
\hline Média (DP) & $4,40(5,39)$ & $4,54(8,52)$ & $0,010^{*}$ \\
\hline Mínimo- Máximo & $0-27$ & $0-36$ & \\
\hline
\end{tabular}

Fonte: BISPO JF, et al., 2021. Legenda: * $p$-valor dos testes qui-quadrado para comparação de proporção.

Ao analisar os problemas clínicos referidos pelos participantes, observou-se que os mais recorrentes entre as mulheres foram: no sistema musculoesquelético (55,8\%), no sistema circulatório e cardiovascular $(18,8 \%)$ e no sistema geniturinário $(14,3 \%)$. Já nos homens, houve um predomínio de problemas no sistema cardiovascular (19,6\%), no sistema musculoesquelético (11,7\%), no sistema respiratório e em processos alérgicos (5,9\%), e no sistema hormonal e endócrino (5,9\%) (Gráfico 1).

Gráfico 1 - Comparação dos problemas clínicos entre mulheres e homens privados de liberdade em duas unidades prisionais de Alagoas - $2020(n=128)$.

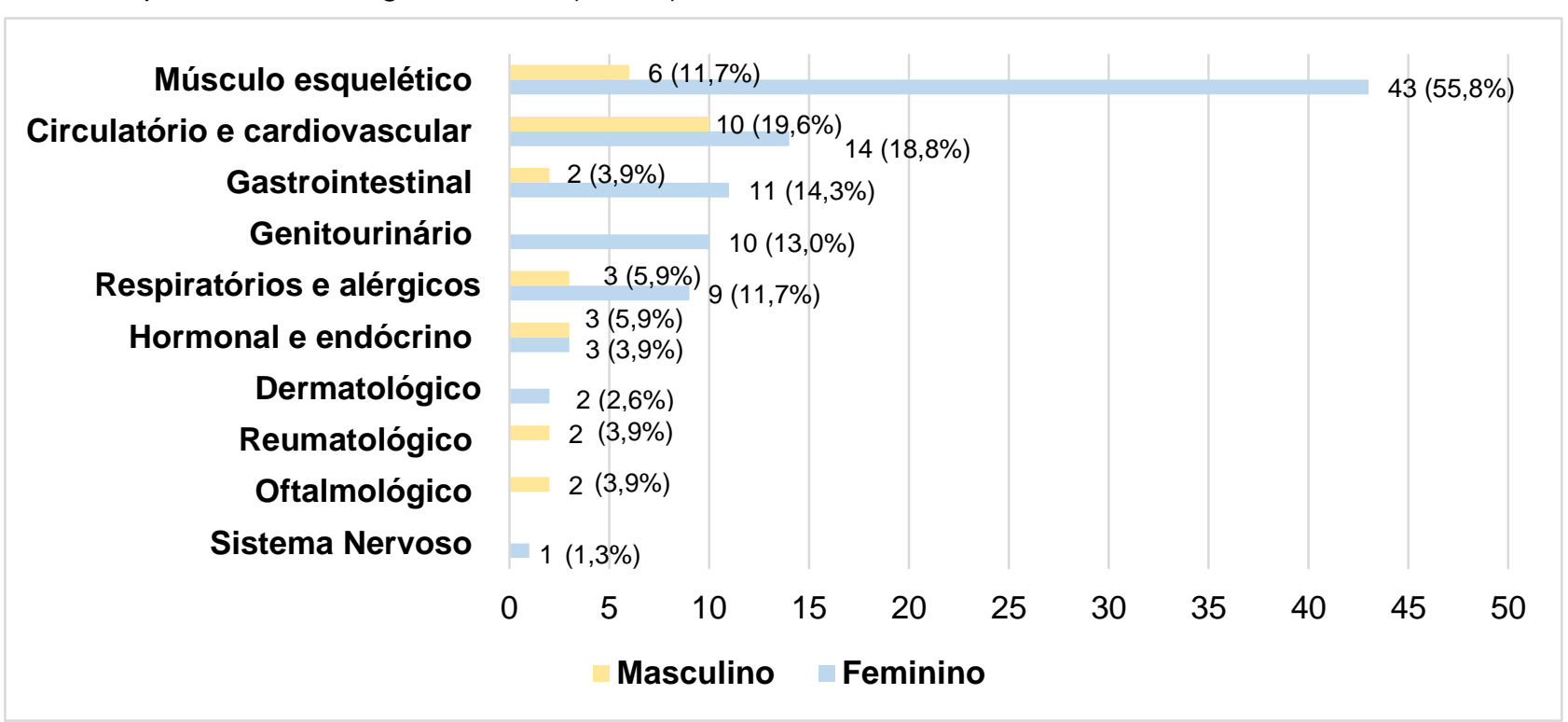

Fonte: Bispo JF et al., 2021.

Observou-se que as queixas de saúde mental mais frequentes entre homens e mulheres foram ansiedade, insônia e depressão. As mulheres apresentaram maior frequência dessas quando comparadas aos homens, com $24,7 \%$ de ansiedade, $15,6 \%$ de insônia e depressão vs. $13,7 \%$ de ansiedade e insônia, e $2 \%$ de depressão, respectivamente (Gráfico 2). 
Gráfico 2 - Comparação das queixas de saúde mental entre mulheres e homens privados de liberdade em duas unidades prisionais de Alagoas - $2020(n=128)$.

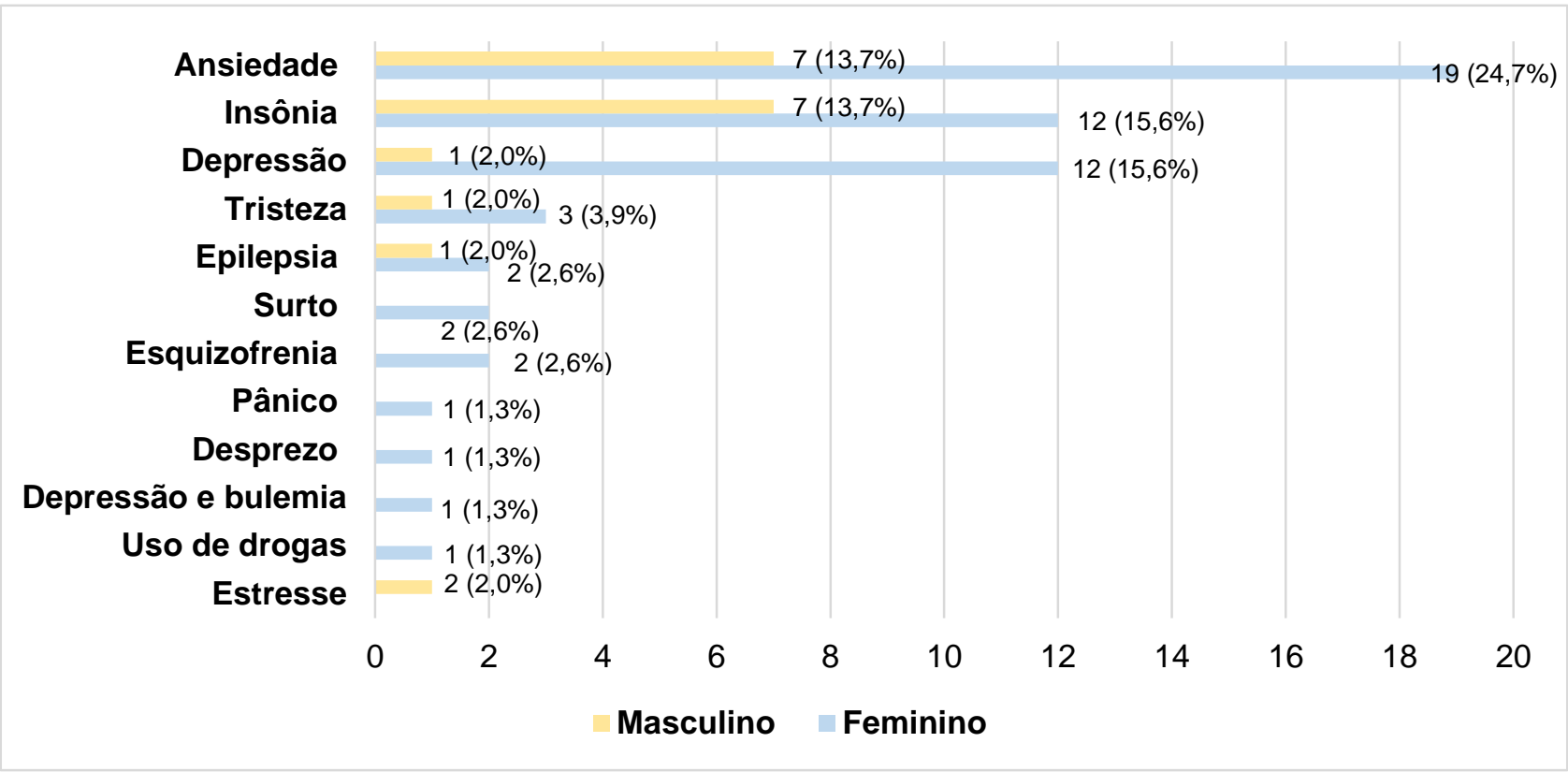

Fonte: Bispo JF et al., 2021.

Ao verificar o uso atual de psicofármacos, observou-se que os ansiolíticos/benzodiazepínicos foram os mais utilizados por mulheres e homens: $42,2 \%$ e $27,5 \%$, respectivamente, acompanhados pelo uso de antidepressivos: $35,5 \%$ das mulheres e $13,7 \%$ dos homens (Gráfico 3 ).

Gráfico 3 - Comparação da prevalência do uso de psicofármacos entre mulheres e homens privados de liberdade em duas unidades prisionais de Alagoas - $2020(n=128)$.

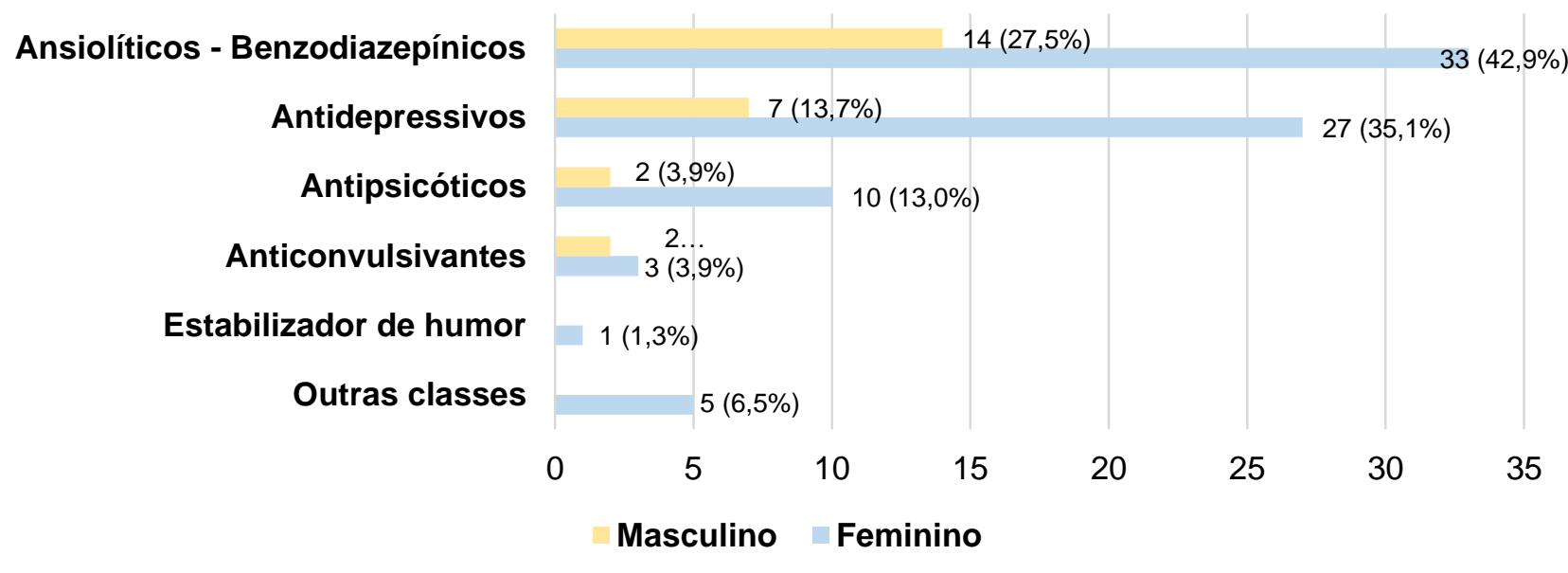

Fonte: Bispo JF et al., 2021.

\section{DISCUSSÃO}

A frequência de problemas clínicos e de saúde mental em mulheres e homens privados de liberdade foi significativa. Ambos relataram problemas clínicos, queixas de saúde mental e uso de psicofármacos no período do encarceramento, bem como possuíam histórico de tratamento de saúde mental. Contudo, o sexo feminino demonstrou maior acometimento dessas condições.

Neste estudo, as queixas/problemas clínicos referidas por ambos os sexos estão relacionados aos sistemas musculoesquelético, circulatório e cardiovascular, corroborando com o estudo de Alves JP, et al. 
(2017), o qual apontou que a população prisional de um município de médio porte do Brasil apresentou prevalência geral, autorrelatada, de $68,7 \%$ de suspeita de alguma doença clínica, em homens e mulheres. Esse percentual teve uma relação direta com as precárias condições de higiene das acomodações.

A assistência à saúde no sistema penitenciário ainda é considerada precária, visto que os cuidados são ofertados de forma limitada, buscando reduzir apenas os danos dos problemas mais emergentes, e desconsiderando a complexidade de outros, o que resulta em adversidades para o acesso de qualidade à saúde (NETO FJ, et al., 2019; SANTANA JCB e REIS FCA, 2019). Em consequência, surgem diversos problemas clínicos, entre eles os cardiovasculares e os relacionados ao sistema musculoesquelético.

Segundo o panorama de saúde da população mundial de 2016, 17,9 milhões de pessoas tiveram como causa de morte as doenças cardiovasculares (WORLD HEALTH ORGANIZATION, 2020). Corroboram, assim, este estudo, o qual constatou que as doenças cardiovasculares são as mais prevalentes entre homens e mulheres privados de liberdade. Hipertensão arterial, transtornos mentais e distúrbios gastrointestinais também são mais frequentes entre as pessoas privadas de liberdade (ALVES JP, et al., 2017).

Observou-se, neste estudo, que a prevalência de tratamento de saúde mental entre as mulheres é duas vezes mais frequente em relação aos homens, bem como a maior prevalência do uso de psicofármacos. Os ansiolíticos/benzodiazepínicos foram os mais utilizados por ambos, com maior prevalência entre as mulheres.

Ressalta-se que há associação entre história de encarceramento, envolvimento com crimes e violência e o surgimento de transtornos mentais (ALTINTAS M e BILICI M, 2018). Ser mulher é um fator de maior suscetibilidade para o surgimento desses transtornos, em decorrência das diferenças de gênero e do papel historicamente associado à mulher (SANTOS LS e DINIZ GRS, 2018). Constata-se neste estudo que as mulheres privadas de liberdade apresentam maior prevalência de tratamento de saúde mental quando comparadas aos homens, bem como maior percentual de sintomas de ansiedade e depressão.

De acordo com o relatório da World Health Organization (2017), em 2015, 264 milhões (3,6\%) de pessoas no mundo possuíam ansiedade. No Brasil, a doença afetava 9,3\% (18.657.934) dos indivíduos. No mundo, 322 milhões $(4,4 \%)$ apresentavam diagnóstico de depressão, enquanto no Brasil a prevalência era de 5,8\%, ou seja, 11.548.577 indivíduos. No presente estudo, a prevalência de sintomas de depressão $(15,6 \%$ nas mulheres e $2 \%$ nos homens) e ansiedade ( $24,7 \%$ nas mulheres e $13,7 \%$ nos homens) foi mais elevada do que na população mundial e no Brasil.

Estar em privação de liberdade pode afetar diretamente a saúde mental desta população. A situação de encarceramento e as condições das instalações aumentam o risco de apresentar depressão e insatisfação com a vida (YI Y, et al., 2017). Ter uma menor oportunidade de trabalho e educação neste ambiente também é um risco para o surgimento de transtornos mentais. Gabrysch C, et al. (2019) evidenciou em seu estudo que trabalhar com remuneração ou estudar durante o encarceramento mostrou uma forte associação com a melhora dos sintomas psicológicos. Já ter sido exposto a abuso ou violência durante a infância aumenta em duas vezes o risco de apresentar depressão, ansiedade e/ou sintomas estressantes graves, pelo menos durante a prisão (SÁNCHEZ FC, et al., 2019).

O relatório da World Health Organization (2017) demonstrou que a depressão e a ansiedade são mais comuns nas mulheres do que nos homens, com um percentual de $5,1 \%$ versus $3,6 \%$ e $4,6 \%$ versus $2,6 \%$, respectivamente. Outros estudos também evidenciaram uma maior taxa de depressão e ansiedade em mulheres, quando comparadas aos homens (KARLSSON ME, et al., 2018; SANTOS MM, et al., 2019). Esses achados corroboram este estudo.

Há fatores físicos, ambientais, sociais e emocionais que podem identificar as causas relacionadas à depressão e à ansiedade entre homens e mulheres, entre os quais as variações hormonais que a mulher sofre ao longo da vida, com relação ao humor e ao comportamento (SENICATO C, et al., 2018). Apesar da escassez de evidências na literatura, estudos mostraram que as mulheres tendem a ser mais vulneráveis aos efeitos depressogênicos da inflamação do que os homens, devido a uma maior exposição ao estresse crônico, o que provoca déficits afetivos e comportamentais causados por alterações neuroimunes, como a ativação 
das células imunes do cérebro, a expressão de citocinas pró-inflamatórias e as alterações na neurotransmissão e na plasticidade sináptica nos circuitos neurais relacionados ao estresse (BEKHBAT M e NEIGH GN, 2017).

No que se refere ao ambiente prisional, há repercussões na saúde mental para ambos os sexos, contudo, nas mulheres isso se torna mais grave, em decorrência da desigualdade social enraizada na sociedade, incluindo a desigualdade de gênero e a financeira (YU S, 2018). Vários fatores negativos, inseridos no ambiente prisional somados à experiência do encarceramento feminino que notoriamente é demarcada pelo abandono familiar, separação dos filhos e de julgamento social, contribuem no maior adoecimento físico e mental das mesmas (SANTOS MV, et al., 2017).

Em relação ao uso de psicofármacos, o percentual entre as mulheres privadas de liberdade foi de $55,8 \%$. De acordo com o estudo de Pontes CAC e Silveira LC (2017), no Brasil, a população feminina consome cerca de duas a três vezes mais psicofármacos, quando comparada à masculina. Tal aspecto pode ser explicado pelas mulheres serem propensas a cuidarem mais de sua saúde do que os homens, o que implica em uma maior procura pelos serviços de saúde, além disso, também pode ser pelo fato de elas serem mais afetadas por conflitos sociais e familiares, buscando utilizar esse tipo de medicamento como um escape aos seus problemas.

Quando essas mulheres se encontram em situação de privação de liberdade, isso se torna mais preocupante, pois a reclusão impacta negativamente na saúde mental e, consequentemente, as mulheres começam a fazer uso de psicofármacos, sobretudo ansiolíticos e benzodiazepínicos, como forma de diminuir esses danos (MINISTÉRIO DA JUSTIÇA, 2019).

É importante enfatizar que as unidades nos quais as coletas do estudo foram realizadas dispõem de diferentes particularidades. A unidade masculina destaca-se por oferecer mecanismos que promovem a ressocialização, tais como instalações com melhores condições e o incentivo e acesso ao trabalho, educação e lazer. Enquanto que na unidade prisional feminina as instalações e atividades apresentam características mais tradicionais, ocasionando em menos oportunidades de trabalho e educação (SERIS, 2018).

Por se acharem num ambiente mais precário, as mulheres tornam-se mais vulneráveis em diversos aspectos. Estes aspectos foram evidenciados no estudo prévio acerca dos fatoressociodemográficos da população do presente estudo, o qual identificou que essas mulheres acumulavam fatores mais desfavoráveis, como renda inferior à dos homens, menor grau de escolaridade e menor acesso ao trabalho remunerado e à educação no período de encarceramento. Além disso, elas tinham relações familiares mais desfavoráveis, incluindo menor número de visitas familiares e maior rompimento de relações conjugais que os homens (PEDROSA TMM et al., 2020).

Os resultados do presente estudo demonstraram que a saúde física e mental de homens e mulheres privados de liberdade necessitam de uma maior atenção dos profissionais de saúde, sobretudo a saúde das mulheres privadas de liberdade, por se encontrarem em condições de maior vulnerabilidade. É necessário refletir sobre a importância do fortalecimento de políticas que buscam extinguir as desigualdades de gênero, assim como propor e instituir condições mais igualitárias no ambiente prisional de acordo com cada especificidade. A existência de melhores possibilidades de ressocialização refletirá na saúde dessa população.

\section{CONCLUSÃO}

No presente estudo, as mulheres foram mais acometidas por problemas físicos e mentais do que os homens. Espera-se que os resultados deste estudo possam contribuir para a proposição e a efetivação de políticas públicas já existentes, incluindo a ampliação e a qualificação do acesso às ações de saúde no sistema prisional. Ressalta-se a necessidade da observância das diferenças de gênero, para garantir a implementação de ações que visam à equidade e à redução dessas desigualdades, com vista à dignidade humana. São necessários mais estudos para analisar as diferenças dos perfis de saúde física e mental de homens e mulheres em situação de privação de liberdade. 


\section{AGRADECIMENTOS E FINANCIAMENTO}

Agradecemos ao Serviço de Administração Penitenciária de Alagoas/Brasil e aos profissionais que atuam no Sistema Prisional pela colaboração para a realização deste estudo. Especialmente, agradecemos aos participantes do estudo, que prontamente aceitaram ser entrevistados e compartilhar características tão singulares das suas vidas. Também agradecemos ao Centro Universitário Tiradentes/Alagoas pelo incentivo e apoio durante o desenvolvimento do estudo no Programa de Iniciação Científica.

\section{REFERÊNCIAS}

1. ALTINTAS M, BILICI M. Evaluation of childhood trauma with respect to criminal behavior, dissociative experiences, adverse family experiences and psychiatric backgrounds among prison inmates. Compr Psychiatry, 2018; 82: $100-107$.

2. ALVES JP, et al. Perfil epidemiológico de pessoas privadas de liberdade. Revista de Enfermagem UFPE online, 2017; 11(10): 4036-4044.

3. BASSANI L, LUCAS CL. Mulheres no cárcere: uma breve análise da situação brasileira. XXV seminário de iniciação científica, 2017.

4. BEKHBAT M, NEIGH GN. Sex differences in the neuro-immune consequences of stress: Focus on depression and anxiety. Brain Behavlmmun., 2018; 67: 1-12.

5. GABRYSCH C, et al. Mental disorders and mental health symptoms during imprisonment: A three-year follow-up study. PLoS One, 2019; 14(3): e0213711.

6. GALVÃO MCB, DAVIM RMB. Ausência de assistência à gestante em situação de cárcere penitenciário. Cogitare Enfermagem, 2013; 18(3): 452-459.

7. GOTTFRIED ED, CHRISTOPHER SC. Mental Disorders Among Criminal Offenders. Journal of Correctional Health Care, 2017; 23(3): 336-346.

8. HENRY BF. Typologies of adversity in childhood \& adulthood as determinants of mental health \& substance use disorders of adults incarcerated in US prisons. Child Abuse \& Neglect, 2020; 99: 1-9.

9. HUGHES MH, et al. Gender Differences in Health Care Needs and Service Attainment Among Violent Offenders. Journal of Correctional Health Care, 2020; 6(1); 55-65.

10. KARLSSON ME, et al. Sexual Victimization and Mental Illness Prevalence Rates Among Incarcerated Women: A Literature Review. Trauma, Violence, \& Abuse, 2018; 21(2): 326-349.

11. KOLLING GJ, et al. O Direito à Saúde no Sistema Prisional. Tempus Actas de Saúde Coletiva, 2013; 7(1): $282-297$.

12. MINAYO MCS, RIBEIRO AP. Condições de saúde dos presos do estado do Rio de Janeiro, Brasil. Ciência e Saúde coletiva, 2016; 21: 2031-2040.

13. MINISTÉRIO DA JUSTIÇA. INFOPEN - Levantamento Nacional de informações penitenciárias. Brasil, 2014. Disponível em: http://www.justica.gov.br/noticias/mj-divulgara-novo-relatorio-do-infopen-nesta-terca-feira/relatoriodepen-versao-web.pdf. Acesso em: 06 jan. 2020.

14. MINISTÉRIO DA JUSTIÇA. INFOPEN - Levantamento Nacional de informações penitenciárias. Brasil, 2019. Disponível em: http://depen.gov.br/DEPEN/depen/sisdepen/infopen. Acesso em: 07 ago. 2020.

15. MINISTÉRIO DA JUSTIÇA. INFOPEN - Levantamento Nacional de informações penitenciárias. Brasil, 2020. Disponível em: https://www.gov.br/depen/pt-br/sisdepen. Acesso em: 10 jan. 2021.

16. MINISTÉRIO DA SAÚDE. Resolução nำ 466, de 12 de dezembro de 2012. Dispõe sobre diretrizes e normas regulamentadoras de pesquisas envolvendo seres humanos. Brasília: Diário Oficial da República Federativa do Brasil. Brasil, 2012. Disponível em: https://bvsms.saude.gov.br/bvs/saudelegis/cns/2013/res0466_12_12_2012.html. Acesso em: 11 jul. 2020.

17. MINISTÉRIO DA SAÚDE. Política Nacional de Atenção Integral à Saúde das Pessoas Privadas de Liberdade no Sistema Prisional. 1. ed. - Brasília: Ministério da Saúde, 2014. Disponível em: http://www.as.saude.ms.gov.br/wpcontent/uploads/2016/06/Cartilha-PNAISP.pdf. Acesso em: 11 jul. 2020.

18. NETO FJ, et al. Health morbidity in Brazilian prisons: a time trends study from national databases. BMJ Open, 2019; 9(5): 1-8.

19. OLIVEIRA KRV, et al. Comportamentos de saúde nas experiências sexuais de mulheres em situação de cárcere. Revista Brasileira Enfermagem, 2019; 72(3): 95-102.

20. PEDROSA TMM, et al. Perfil sociodemográfico de homens e mulheres privados de liberdade: um estudo comparativo. International Journal of Development Research, 2020; 10(10): 41713-41720.

21. PEREIRA WRM, FROTA AS. Assistência à saúde dos reeducandos na Unidade Prisional Avançada de São José do Cedro -SC. Anuário Pesquisa e Extensão Unoesc São Miguel do Oeste, 5, e24559.

22. PONTES CAC, SILVEIRA LC. Abuso de benzodiazepínicos entre mulheres: o que esse fenômeno (re)vela? Revista de políticas públicas, 2017; 16(1): 15-23. 
23. RODRIGUES MIL, CORDAZZO K. Os atuais problemas carcerários: uma análise crítica da superlotação carcerária e a ineficiência do estado. Revista Reflexão e Crítica do Direito, 2019; 7(2): 203-223.

24. SÁNCHEZ FC, et al. Associations between childhood abuse, mental health problems, and suicide risk among male prison populations in Spain. Criminal BehaviourAnd Mental Health, 2019; 29(1): 18-30.

25. SANTANA JCB, REIS FCA. Percepção da equipe de enfermagem acerca da assistência à saúde no sistema prisional. Revista de Pesquisa: Cuidado é Fundamental Online, 2019; 11(5): 1142-1147.

26. SANTOS LS, DINIZ GRS. Saúde mental de mulheres donas de casa: um olhar feminista-fenomenológico-existencial. Psic. Clin., 2018; 30(1): 37-59.

27. SANTOS MM, et al. Fatores correlatos de depressão entre internos do sexo masculino e feminino. Rev. bras. epidemiol., 2019; 22: e190051.

28. SANTOS MV, et al. Mental health of incarcerated women in the state of Rio de Janeiro. Texto \& Contexto Enfermagem, 2017; 26(2): e5980015.

29. SECRETARIA DE ESTADO DE RESSOCIALIZAÇÃO E INCLUSÃO SOCIAL (SERIS). In: Unidades do Sistema Prisional Alagoano. Alagoas, 2018. Disponível em: http://www.seris.al.gov.br/unidades-do-sistema. Acesso em: 24 fev. 2020.

30. SENA EP, et al. Irismar: psicofarmacologia clínica. 3.ed. Rio de Janeiro: MedBook, 2011.

31. SENICATO C, et al. Transtorno mental comum em mulheres adultas: identificando os segmentos mais vulneráveis. Ciênc. saúde coletiva, 2018; 23(8): 2543-2554.

32. WORLD HEALTH ORGANIZATION (WHO). Depression and Other Common Mental Disorders: Global Health Estimates. Geneva: World Health Organization; 2017. Other Common Mental Disorders: Global Health Estimates. Geneva: World Health $2017 . \quad$ Disponível em: https://apps.who.int/iris/bitstream/handle/10665/254610/WHO-MSD-MER2017.2eng.pdf;jsessionid=062F0E14AD0C197E6BCCC98765FC4099?sequence=1. Acesso em: 07 ago. 2020.

33. WORLD HEALTH ORGANIZATION (WHO). World health statistics 2020: monitoring health for the SDGs, sustainable development goals. Geneva: WHO; $2020 . \quad$ Disponível em: https://apps.who.int/iris/bitstream/handle/10665/332070/9789240005105-eng.pdf?ua=1. Acesso em: 10 ago. 2020.

34. WORLD PRISON BRIEF. World Prison Population List. 12 ed. 2018. Disponível em: https://www.prisonstudies.org/. Acesso em: 10 ago. 2020.

35. YI Y, et al. Mental Health Among Jail and Prison Inmates. Am J Mens Health, 2017; 11(4): 900-909.

36. YU S. Uncovering the hidden impacts of inequality on mental health: a global study. Translational Psychiatry, 2018; $8(98)$. 